\title{
Stress state and power parameters during pulling workpieces through a special die with an inclined working surface
}

\author{
Zhassulan Ashkeyeva $^{a}$, Maksat Abishkenov ${ }^{\text {* }}$, Serik Mashekov ${ }^{\mathrm{c}}$ and Anna Kawalek ${ }^{\mathrm{d}}$
}

${ }^{a}$ Candidate of Engineering Sciences, Associate Professor, Department of Metal Forming, Karaganda Industrial University, 30 Republic Ave., 101400 Temirtau, Kazakhstan

${ }^{b}$ Master of Science, Doctoral Student, Department of Metal Forming, Karaganda Industrial University, 30 Republic Ave., 101400 Temirtau, Kazakhstan

${ }^{c}$ Doctor of Science, Full Professor, Department of Industrial Engineering, Satbayev University, 22 a Satbayev Str., 050013 Almaty, Kazakhstan

${ }^{d}$ Doctor of Science, Full Professor, Department of Metal Forming and Safety Engineering, Czestochowa University of Technology, 69 Dabrowskiego Str., 42-201 Czestochowa, Poland

\begin{tabular}{l}
\hline A R T I C L E I N F O \\
\hline Article history: \\
Received 20 October 2020 \\
Accepted 30 November 2020 \\
Available online \\
5 December 2020 \\
\hline Keywords: \\
Pulling \\
Special die \\
Shear angle \\
Shear strain \\
Stress state \\
Slip line field
\end{tabular}

\begin{abstract}
A B S T R A C T
The study of the stress state and power parameters when pulling workpieces in a special die with an inclined working surface at various shapes of the plastic deformation zone and geometric parameters of the special die was conducted. The distinctive feature of the proposed special die and the metal treatment process in the working channel of this die was described. The theoretical provisions and assumptions from the fundamental theory of plasticity and metal forming were used. The influence of the intensity of shear deformations on the stress state and force at the angles of inclination of the working surface of the die within $45-20^{\circ}$, the value of the ratio of the diameter of the workpiece to the length of the inclined surface $\mathrm{d} / \mathrm{z}=1.5-2.0$ was investigated. The optimal $\mathrm{d} / \mathrm{z}$ ratio was determined by the method to a rigid punch indenting a rigid-plastic half-space, as well as by the method of strain energy. The field of slip lines and hodographs of velocities for various shapes of the deformation zone and geometric parameters of a special die were constructed. Based on the constructed slip line fields and velocity hodographs, the mean stress and stress components at the nodal points of the slip line field with the compilation of the equilibrium equations for all forces applied to the plastic zone were calculated. The study of the influence of contact friction between the working surface of the die channel and the workpiece on the stress state and power parameters during pulling was carried out. It was revealed that the optimal ratio $\mathrm{d} / \mathrm{z}=1.5$ and the optimal angle of inclination of the working channel of the die $\alpha=20^{\circ}$. It was found that for these parameters in the zone of plastic deformation, mainly significant compressive stresses act, which favorably affect the obtaining of a homogeneous and refined microstructure, and also exclude the appearance of anisotropy due to the implementation of maximum shear deformations in the workpiece.
\end{abstract}

(c) 2021 Growing Science Ltd. All rights reserved.

\section{Introduction}

As it is known, for metal products, in particular for long metal workpieces and products made from them (wires, bars, rods), increased requirements are imposed on physical and mechanical properties (strength, wear resistance, plasticity, electrical conductivity etc.). One of the effective ways to ensure the production of high-quality long workpieces is an intensive elaboration of the material structure by intensifying plastic deformation, the main of which are various versions of the severe plastic deformation * Corresponding author. Tel.: +77084934863

E-mail addresses: maks91.kz@inbox.ru (M. Abishkenov) 
processes (Valiev et al., 2014; Azushima et al., 2008; Zehetbauer \& Valiev, 2004; Ghader et al., 2018; Harsha et al., 2018; Segal, 1999).

The process of pulling workpieces in the known conical dies (drawing dies) with a approach zone and a bearing zone (Wright, 2016) leads to the stretching of grains in the direction of pulling. In the known dies (drawing dies), the grains are mainly stretched in the longitudinal direction, i.e. in the direction of pulling, and in the transverse direction, the flow of the metal is insignificant, which leads to a difference in mechanical properties - anisotropy throughout the volume of the material (Iordachescu et al., 2015; Toribio, 2019; Zolotorevsky \& Krivonosova, 1996; Rajan \& Petkie, 1998; Carlsson \& Huml, 1996). Therefore, it is of great interest to change the direction of the metal flow due to the action of tools with a special configuration of the working surface, which ensures the equalization of mechanical properties throughout the volume of the material. In addition, during intensive stretching, dangerous tensile stresses appear which can lead to failure when the material reaches its yield point. Therefore, the development of new ways of pulling and special tools for its implementation is the most urgent task.

This work proposes the design of a special die with an inclined working surface for pulling a cylindrical workpiece with a diameter $d$. The die consists of two semi-dies fastened together by fasteners or enclosed in a common cage (Fig. 1).

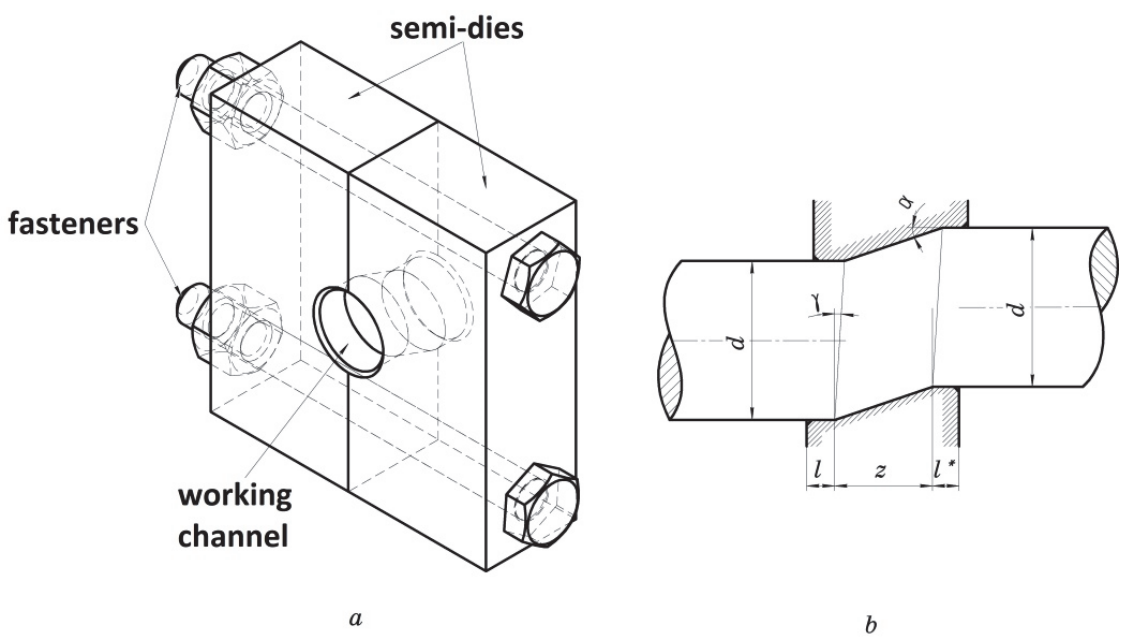

Fig. 1. Design (a) and parameters of the working channel (b) of a special die with an inclined working surface ( $\alpha$ - the shear angle, $\gamma$ - the angle of displacement of the working surfaces of the die, $d$ - the diameter of the pulled workpiece, $z$ - the length of the shear section, $l$ - the length of the entrance zone, $l^{*}$ - length of the bearing zone).

A distinctive feature of the design of a proposed special die and the metal forming process in the working channel of this die is that the unidirectional metal flow is disturbed in the shear or transfer section $z$, which in turn leads to a more uniform distribution of mechanical properties throughout the workpiece volume. In addition, the design of a special die, preventing excessive stretching of grains, contributes to the closure and brewing (elimination) of all internal defects (porosity, bubbles, etc.), and also reduces the likelihood of cracking in the longitudinal direction of pulling workpieces by reducing the most dangerous tensile stresses

A special die can be installed as bar and section mills, wire mills after the billet or bar leaves the passes of the mill rolls or the hole of the drawing die for additional (intensive) processing of the metal and to prevent excessive stretching of grains. As part of an experimental study, it is planned to use this die as part of a sequential technological cycle, which includes other scientific developments of authors (Abishkenov et al., 2020; Mashekov et al., 2020; Ashkeev et al., 2005; Naizabekov et al., 2010). 


\section{Investigation of the stress-strain state and power parameters for various shapes of the plastic deformation zone and geometric parameters of a special die}

\subsection{Basic theoretical principles and assumptions}

The intensity of metal working in the proposed die depends on the angle of inclination or shear angle $\alpha$ of the die and the ratio of the diameter of the workpiece $d$ to the length of the shear section $z$. From the fundamental theory of plasticity and metal forming (Hosford, 2013; Doltsinis, 2001; Dixit \& Dixit, 2014; Chung \& Lee, 2018), it is known that the greater the value of the shear angle $\alpha$, the greater the intensity of the working out, but the greater the required pulling force, and vice versa, the smaller the angle $\alpha$, the lower the pulling force and the degree of metal working. It is also known from the theory of plastic deformation of metals that at large shear deformations due to large values of $\alpha$, the body can be destroyed, which is called a shearing or a shear fracture. This means that in the design of tools for the implementation of plastic deformation of metal, it is especially important to prevent the destruction of the metal (shearing) and the selection of the optimal parameters of the tool, which will give the best effect in achieving optimal strength characteristics. Therefore, the main task of the work is to study the stress-strain state (SSS) and power parameters at various ratios $d / z$ and values of the shear angle $\alpha$, in particular, at $\alpha=20^{\circ}, \alpha=30^{\circ}$ and $\alpha=45^{\circ}$ in order to determine the optimal values these parameters, under different conditions of deformation, i.e. at different values of the coefficient of contact friction $f$.

The influence of the intensity of shear strains on the quality of the metal is described in many works, in particular in (Frint \& Wagner, 2019; Goloujeh \& Soltanpour, 2020; Rahimi et al., 2017), where the required quality of the metal is provided due to the alternating shear strain, but the effect on the nature of the metal flow during its strain or deformation is not mentioned. To study the influence of the intensity of shear strain on the SSS and the force, the angles of inclination of the working surface of the die $\alpha$ were chosen in the range of $45-20^{\circ}$, as well as at $15^{\circ}$, and the value of the ratio $d / z=1.5-2.0$. The selected interval is explained by the fact that, at $\alpha \geq 45^{\circ}$ and $d / z>2.0$, in addition to an increase in the pulling force, it is possible that the workpiece will be indented into the upper protrusion of the die or the workpiece will be shear fracture in the shear section when processing low-plastic, brittle materials. In addition, with an increase in the angle of inclination $\alpha$, the total pulling force increases, which can lead to slip of the rolling mill rolls, after when the workpiece enters the working channel of the die or breakage of the workpiece itself. Therefore, the determination of the optimal value of the ratio $d / z$ and the angle of inclination $\alpha$ is the most important in the development of technology for pulling or drawing long workpieces.

First of all, it's necessary to determine the optimal value of the $d / z$ ratio, at which the maximum shear of the most plastic materials without indention and low-plastic materials without shearing fracture on the inclined section of the die can occur. The indention of the protrusion of the die into the workpiece or the shearing fracture of a low-plastic material is possible at large values of the $d / z$ ratio, i.e. for large shapes of the plastic deformation zone, where $d$ is the diameter or the maximum size of the pulled workpiece, $z$ is the section of shear of one part of the workpiece relative to another, or the section of metal transfer. It should be noted that at large values of the $d / z$ ratio, the shear, and hence the elaboration of the structure, may not occur. The reason for this phenomenon is that the shear strength $P_{s h}$ will be so significant that the body will be forced to indent into the upper protrusion of the die when processing plastic materials, or shearing can occur when processing low-plastic materials. Therefore, the first main task is to determine the optimal value of the $d / z$ ratio when pulling through a special die with an inclined working surface. To solve this issue, we use the basic equations from the theory of plasticity, in particular the method of slip lines (s.l.) or characteristics, which have proven themselves well in solving plane and axisymmetric problems and other computational and analytical methods (Hosford, 2013; Hill, 1998; Yu et al., 2006; Bhaduri, 2006; Rees, 2006). 


\subsubsection{Determination of the optimal value of the $d / z$ ratio when pulling low-plastic materials}

The condition under which the maximum shear is possible without indenting the workpiece into the protrusion of the die can be represented as follows: $P_{s h}<P_{i n}$, where $P_{s h}$ is the force necessary to overcome the resistance (shear strength) or shear force on the inclined section of the working channel of the die, $P_{\text {in }}$ is the force required to indent the protrusion of the workpiece working channel into the workpiece. To determine the force of indention $P_{i n}$, we use the method of indenting the protrusion of the die into the plastic half-space (Hill, 1998; Lee \& Kobayashi, 1970; Hardy et al., 1971; Voyiadjis \& Buckner, 1983). The field of s.l. when the upper trapezoidal protrusion of the die is indented into the workpiece is shown in Fig. 2.

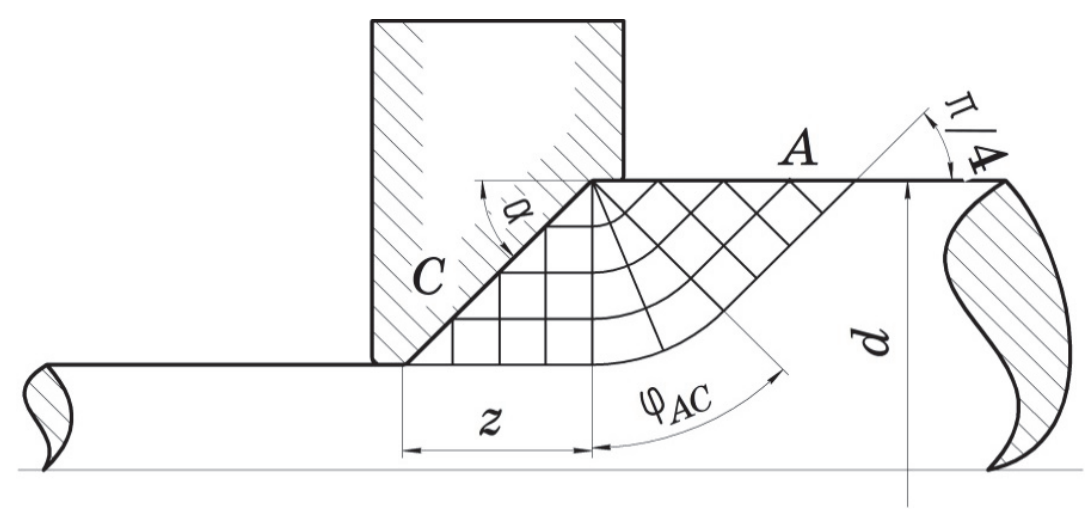

Fig. 2. The field of slip lines when the trapezoidal protrusion of the die is indented into the plastic half-space

The difference lies in the fact that in this case, the trapezoidal protrusion of the die is indented into the workpiece, where the angle of rotation of s.l. $\varphi_{A C}=45^{\circ}$ or $\pi / 4$ radians (Fig. 2). Then the specific indention force of the protrusion of the die into the workpiece will be:

$$
P_{\text {in }}=2 k\left(1+\varphi_{A C}\right)=2 k\left(1+\frac{\pi}{4}\right)=2 k \cdot 1.785,
$$

where $k$ is the plastic constant or is the yield stress of the material in shear.

The specific shear force of the workpiece on the inclined section of the die at the first stage of the calculation can be determined using the formula for determining the shear force taking into account plastic bending with some assumption that the shear occurs mainly in the transfer section:

$$
P_{s h}=\sigma_{s h} F_{s h} \omega,
$$

where $\sigma_{s h}$ is shear strength (resistance to shear or shearing), the value of which can be expressed through the strain resistance $\sigma_{S}$, taking into account the correction factor or coefficient of correction 0.8 , i.e. $\sigma_{s h}=$ $0.8 \sigma_{S}=2 k \cdot 0.8 ; F_{s h}=\left(\pi d^{2}\right) / 4$ is shear area for a cylindrical workpiece; $\omega$ is the coefficient taking into account the plastic bending (taken in the range $1.1 \div 1.5$ ). Taking into account the maximum plastic bending, the value of the coefficient $\omega$ is assumed to be 1.5. Then expression (2) takes the following form:

$$
P_{s h}=0.8 \cdot 2 k=\frac{\pi d^{2}}{4} \cdot 1.5
$$


By quating the shear force $P_{s h}$ and the indention force $P_{i n}$ we get:

$$
2 k \cdot 1.785 \cdot 0.5 \pi d z=0.8 \cdot 2 k \cdot \frac{\pi d^{2}}{4} \cdot 1.5,
$$

where $0.5 \pi d z$ is the contact area of the inclined surface of the die and the workpiece, which is determined through the workpiece diameter $d$ and the gap $z$. The value of the coefficient $\omega$ is taken equal to 1.5, taking into account the maximum plastic bending. Transforming the expression $\left(3^{*}\right)$ we get $0.8925 z=$ $0.3 d$. Hence $d / z=2.975 \approx 3.0$, i.e. the value of this ratio should not exceed 3.0, and thus confirms the previously made assumption that at large values of the diameter of the workpiece $d$ in relation to the section $z$, it is practically impossible to carry out a shear. It should be noted that if the gap $z$ is too small, i.e. if the $d / z$ ratio is too high, it is also possible to shearing fracture in the workpiece, accompanied by the separation of one part of the workpiece relative to another, especially when processing low-plasticity metals and alloys.

For small values of the ratio, for example, when $d / z<1.5$, the length of the inclined surface of the die (gap length $z$ ) increases, and, accordingly, the resistance of the forces of contact friction increases, which in turn will lead to an increase in the total pulling force.

\subsubsection{Determination of the optimal value of the $d / z$ ratio when pulling plastic materials}

The optimal $d / z$ ratio in the second case can be determined as in the previous case, equating the indention force $P_{\text {in }}$ and the shear force $P_{s h}$ of the workpiece on the inclined section, which can be expressed through the shear strain energy $A_{s h}$, i.e. according to the formula:

$$
A_{s h}=\sigma_{i} V_{s h} \varepsilon_{i}
$$

where $\sigma_{i}$ is the stress intensity, the value of which during shear can be taken equal to the value of the strain resistance during shear, through the following ratio $k=\sigma_{S} / \sqrt{3} ; V_{s h}$ is the volume or area of the workpiece in a plastic state at the shear area of the die; $\varepsilon i$ is the strain intensity, which can be written in terms of the shear strain intensity $\mathrm{Y}=\tan \alpha$, and the relationship that connects these values, i.e. $\varepsilon_{i}=$ $Y / \sqrt{3}$, hence $\varepsilon_{i}=\sqrt{3} \tan \alpha$. Hence the shear strain energy:

$$
A_{s h}=3 k V_{s h} \tan \alpha=3 k\left(\frac{\pi d^{2}}{4}\right) z \tan \alpha .
$$

The shear force can be determined by dividing the shear strain energy by the shear of the workpiece from the side of the protrusion of the upper die $u$, which can be written through the angle of inclination $\alpha$ and the length of the inclined section $z$, i.e. $u=z \tan \alpha$. Hence the shear force:

$$
P_{s h}=3 k\left(\frac{\pi d^{2}}{4}\right)
$$

As in the previous case, equating the indention force $P_{\text {in }}$ and the shear force $P_{s h}$, we obtain that the optimal value of the $d / z$ ratio is $d / z=2.38$. Comparing with the previously obtained value $(d / z=3)$, we can conclude that the optimal value of the $d / z$ ratio should be in the range $2-3$, but with some margin we accept this ratio in the range $d / z \approx 1.5 \div 2.0$, i.e. e. the obtained value can be considered optimal.

Analyzing the obtained $d / z$ values by two methods, we can draw the following conclusion that the first version of the condition for realizing the maximum shear $(d / z=2.0 \div 3.0)$ is suitable for processing low-plastic and brittle materials, where, at large $d / z$ ratios, in addition to indention (bearing), and shear 
fracture of material. The second condition for the development of maximum shear $(d / z=2.5 \div 3.0)$ is more suitable for plastic materials, where shear is carried out without shear fracture, i.e. due to plastic shear deformation of the workpieces. Therefore, the above ratio $d / z=1.5 \div 2.0$ can be approved as the only correct solution. After determining the required optimal $d / z$ ratio, the assessing the stress state when pulling a round or oval bar through a special die with an inclined working surface was conducted.

\subsection{Study of the stress state when pulling the workpiece through a special die}

\subsubsection{Plotting the field of slip lines and velocities hodograph}

The angle of inclination of the working surface or shear angle $\alpha$ will initially be assumed equal to $20^{\circ}$, and the value of the ratio $d / z=1.5$, where the above condition is satisfied, and the maximum shear is ensured, and not the indention of the tool protrusion into the workpiece and the minimum pulling force is provided. It should be noted that with an increase in the angle of inclination $\alpha$, the total pulling force increases accordingly due to significant frontal resistance. The slip line field and the velocity hodograph on the inclined shear section at $\alpha=20^{\circ}$ and $d / z=1.5$ are shown in Fig. 3.

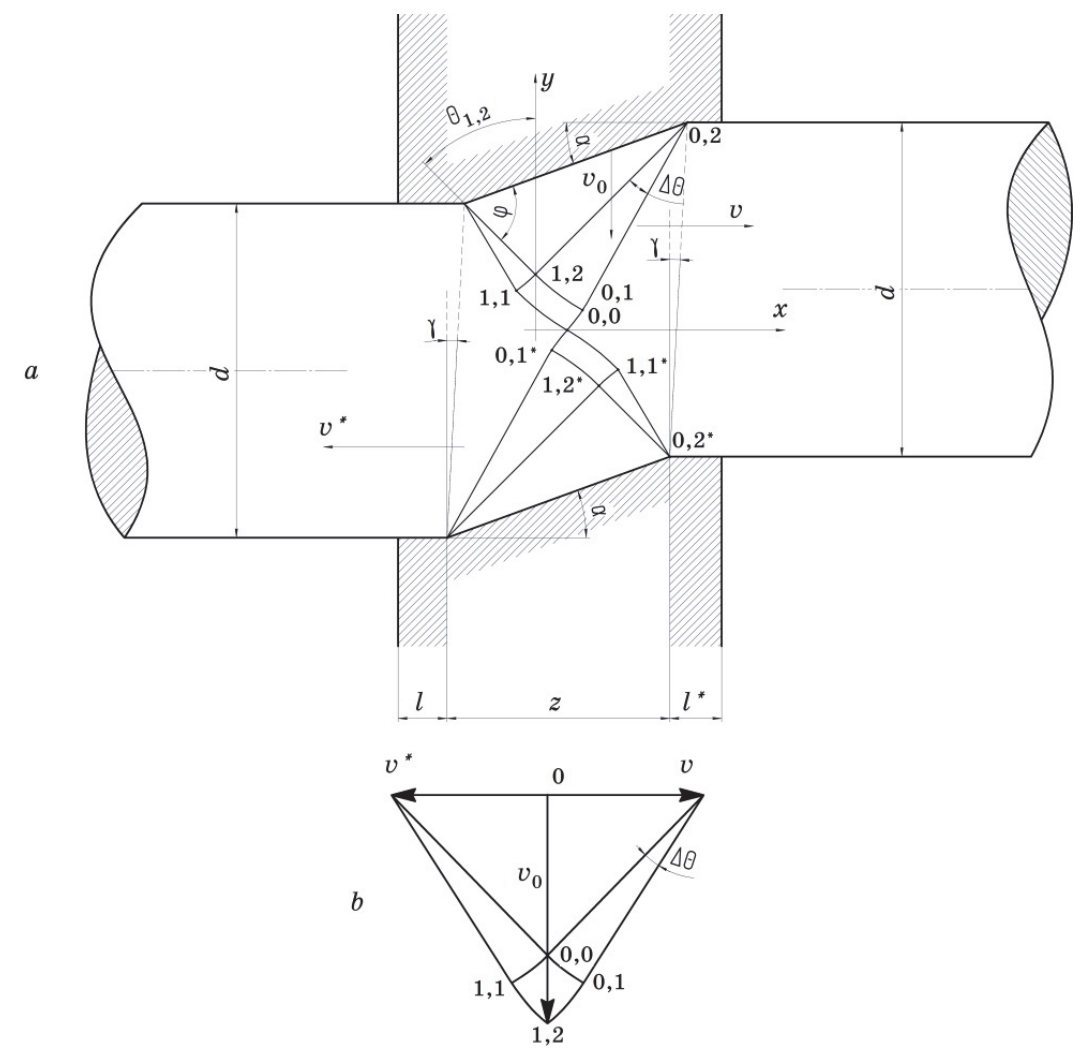

Fig. 3. Slip line field $(a)$ and velocity hodograph $(b)$ on an inclined shear section at $\alpha=20^{\circ}$ and $d / z=1.5$

Theoretically, it can be assumed that an intense metal flow is possible in the direction of the velocities $v$ and $v^{*}$ due to the action on the workpiece from the upper and lower die protrusions. As a result of this action of the tool protrusions, the metal is sheared in the section of the inclined surface or the transfer $z$. In this case, the workpiece receives a shear not only in the transfer section, but also additional shears in the longitudinal direction in the direction of the speeds $v$ and $v^{*}$. For this, the die design provides for a slight displacement of the working surfaces of the die by the angle $\gamma=2-3^{\circ}$. Thus, in the proposed special die, the workpiece receives a shear not only in the transverse direction, but also in the longitudinal direction. The length of the entrance zone $l$ and the bearing zone $l^{*}$ must be taken as minimal as possible, 
since with an increase in the indicated lengths, the frictional resistance force and the total pulling force increase. Field of s.l. drawn from the side of the upper and lower working surface of the die to the intersection with the main $x$-axis, which s.l. must cross at an angle of $45^{\circ}$, which means that the drawn s.l. mesh is correct. (see Fig. 3a). The construction of the field of s.l. and the hodograph of velocities was carried out according to the procedure described in works on the theory of plasticity and metal forming (Hosford, 2013; Hill, 1998; Yu et al., 2006; Bhaduri, 2006; Rees, 2006). At an angle of inclination of the working surface of the die or a shear angle $\alpha=20^{\circ}$, the angle of the s.l. on the contact surface is determined depending on the magnitude of the shear stresses $\tau_{k}$ on the contact surface from the following relation:

$$
\tau_{k}=\frac{\cos 2 \varphi}{2} \sigma_{n}
$$

where the ratio $(\cos 2 \varphi) / 2$ expresses the value of the contact friction coefficient $f$ on the contact surface, i.e. $f=(\cos 2 \varphi) / 2 ; \varphi$ is the outlet angle of s.l. on the contact surface, which can be determined through the value of $f$ and vice versa; $\sigma_{n}$ is the normal stress on the contact surface, which is equal to the yield strength of the processed material. Then from the above ratio, the output angle of s.l. on the contact surface will be $\varphi=25 \div 65^{\circ}$, the friction value on the contact surface will be $f=(\cos 2 \varphi) / 2 \approx 0.321$, i.e. the value of friction is close to the average value, which is quite probable under real conditions of metal forming processes. Geometrically, it is not difficult to establish that the angle of inclination of the s.l. to the $y$ axis at the nodal point 1,2 is $45^{\circ}$, i.e. $\theta_{1,2}=45^{\circ}$. Assume the step of changing the s.l. $\Delta \theta=15^{\circ}$, then adding $\Delta \theta$ to the value $\theta_{1,2}$, we obtain the corresponding value of the angle of inclination of the s.l. to the $y$-axis at the nodal point 0,1 , i.e. the tilt angle will be $\theta_{0,1}=60^{\circ}$, at the nodal point 1,1 will be $\theta_{1,1}=30^{\circ}$, because we subtract $\Delta \theta$ from the value $\theta_{1,2}$. The angle of inclination at the nodal point 0,0 , which is located on the main axis $x$, was carried out according to the formula:

$$
\theta_{i j}=\frac{\theta_{i+1 ; j+1}+\theta_{i ; j+1}}{2}
$$

where $i, j$ are the corresponding indices of the nodal points of the s.l. field, hence

$\theta_{0,0}=\frac{\theta_{1,1}+\theta_{0,1}}{2}=\frac{30^{\circ}+60^{\circ}}{2}=45^{\circ}$.

The intersection of s.l. with the main $x$-axis at an angle of $45^{\circ}$ indicates the correctness of the mesh of s.l. In a similar way, the mesh of s.l. was constructed from the side of the lower working surface of the die. In addition, the correctness of constructing the mesh of s.l. can be checked from the condition of incompressibility of the workpiece when pulling in a special die. For this, a kinematic possible velocity field was constructed (see Fig. 3b), where from the incompressibility condition it can be written:

$\frac{v_{0}}{v}=\frac{d}{Z} \approx 1.5$

where $v_{0}$ and $v$ are the metal flow rates along the $y$ and $x$ axes, respectively, $d$ is the diameter of the bar in the zone of plastic deformation, $z$ is the section of shear of one part of the workpiece relative to another (see Fig. 3, a). Hence, with a certain error of $\sim 1 \div 3 \%$, it can be assumed that the incompressibility condition is fulfilled, that is, the constructed field of s.l. corresponds to the kinematically possible velocity fields under known boundary conditions. Field of s.l. in the shear section is located skewsymmetrically relative to the main $x$-axis (for example, when upsetting between flat frictionless dies, the s.l. fields will be symmetrical). In this case, due to the skew-symmetric arrangement of the working surfaces of the die s.l. field is distorted and transformed, as shown in Fig. 3a. The velocity field in the considered method of pulling in a special die is fundamentally different from the traditional method of 
drawing in conical dies. In the traditional method of pulling or drawing the workpieces, the ratio of speeds is determined, respectively, by the ratio of the areas at the entrance and exit from the zone of plastic deformation of the workpiece. In the traditional pulling method, the workpiece receives a reduction, and the ratio of the speeds depends on the amount of reduction. In the proposed method of pulling in a special die, the workpiece at the entrance and at the exit of the deformation zone does not experience reduction and changes slightly on the inclined section of the die. Plastic deformation occurs only in the shear section (or shear zone). The cross-sectional area of the pulled workpiece or the diameter $d$ after leaving the bearing zone practically does not change and remains equal to the original. Therefore, in accordance with this, the corresponding field of velocities was constructed, relating only to the considered case.

\subsubsection{Calculation of stress components in the plastic deformation zone}

Based on the constructed field of s.l. and the hodograph of velocities, the calculating of mean stress and stress components at the central nodal point $(0,0)$ was performed. To do this, the equilibrium equation for all forces applied to the plastic zone on the right side of the entrance to the plastic deformation zone was described:

$$
\int_{0,0}^{0,1} \sigma d y+\sigma_{0,1}\left(y_{0,2}-y_{0,1}\right)+k x_{0,2}+\int_{1,1^{*}}^{0,0} \sigma^{*} d y+\sigma_{1,1^{*}}\left(y_{1,1^{*}}-y_{0,2^{*}}\right)+k x_{0,2^{*}}=0 .
$$

Using Hencky equations (Dixit \& Dixit, 2014; Hill, 1998; Yu et al., 2006; Rees, 2006), after substitution into the above equation and the corresponding transformation:

$$
\left\{\begin{array}{c}
\sigma=\sigma_{0,0}-2 k\left(\theta-\frac{\pi}{4}\right) \\
\sigma_{0,1}=\sigma_{0,0}-2 k\left(\theta_{0,1}-\frac{\pi}{4}\right) \\
\sigma^{*}=\sigma_{0,0}+2 k\left(\theta^{*}-\frac{\pi}{4}\right) \\
\sigma_{1,1}=\sigma_{0,0}+2 k\left(\theta_{1,1^{*}}-\frac{\pi}{4}\right)
\end{array}\right\},
$$

where $\sigma$ and $\sigma^{*}$ are the mean normal stress along the s.1. is $0,0-0,1$ and $0,0-1,1^{*}$, respectively; $\sigma_{0,1}$ and $\sigma_{1,1 *}$ are mean normal stress at the nodal points 0,1 and $1,1^{*}$, respectively; $\sigma_{0,0}$ is the mean normal stress at the nodal point 0,$0 ; k$ is plastic constant or the yield stress of the material in shear; $\theta$ and $\theta^{*}$ are the angles of inclination of the tangent along the sliding lines $0,0-0,1$ and $0,0-1,1^{*} ; \theta_{0,1}, \theta_{1,1}$ and $\theta_{1,1} *$ are the angles of inclination of the s.l. at the nodal points $0,1,1,1$ and $1,1^{*} ; y_{0,0}, y_{0,1}, y_{1,1}, y_{1,1^{*}}$ and $y_{0,2^{*}}$ are the coordinates of the nodal points $0,0,0,1,1,1,1,1^{*}, 0.2^{*}$ along the $y$-axis, respectively; $x_{0.2}$ and $x_{0.2 *}$ are the coordinates of nodal points 0.2 and $0.2^{*}$ along the $x$-axis. All values are taken directly from Fig. 3 .

Substituting the values of stresses from relations (10) into (9) and transforming the resulting expression, the following equation was obtained:

$$
\frac{\sigma_{0,0}}{2 k}=\frac{\theta y_{0,1}+\theta_{0,1}\left(y_{0,2}-y_{0,1}\right)+\theta^{*} y_{1,1^{*}}-\theta_{1,1^{*}}\left(y_{1,1^{*}}-y_{0,2^{*}}\right)-\frac{\pi}{4}\left(y_{0,2}+y_{0,2^{*}}\right)-x_{0,2}}{y_{0,2}-y_{0,2^{*}}} .
$$

After substituting the values of coordinates and angles directly from Fig. 3, into the last ratio:

$\frac{\sigma_{0,0}}{2 k}=-0.947$ 
The obtained value after substitution into equation (9) completely satisfies it. Stress components at the axial nodal point $(0,0)$ :

- stress along the $y$-axis:

$\sigma_{y_{0,0}}=\sigma_{0,0}-k \sin 2 \cdot \theta_{0,0}=-2 k(0.947+0.5)=-2 k \cdot 1.447$,

- stress along the $x$-axis:

$\sigma_{x_{0,0}}=\sigma_{0,0}+k \sin 2 \cdot \theta_{0,0}=-2 k(0.947-0.5)=-2 k \cdot 0.447$,

- shear stress at the axial nodal point 0,0 :

$\tau_{x y_{0,0}}=-k \cos 2 \cdot \theta_{0,0}=0$.

Next, the stress state when pulling long workpieces through a special die with an angle of inclination $\alpha=30^{\circ}$ was considered. At an output angle of s.l. on the contact surface at an angle $\varphi=30^{\circ}$, respectively, the value of the friction coefficient $f$ is 0.25 . Field of the s.l. and the hodograph of velocities when pulling workpieces with a relatively high shape of the deformation zone with a value of the ratio $d / z \approx 2$ through a die with an angle of inclination of the working surfaces $\alpha=30^{\circ}$ is shown in Fig. 4 .
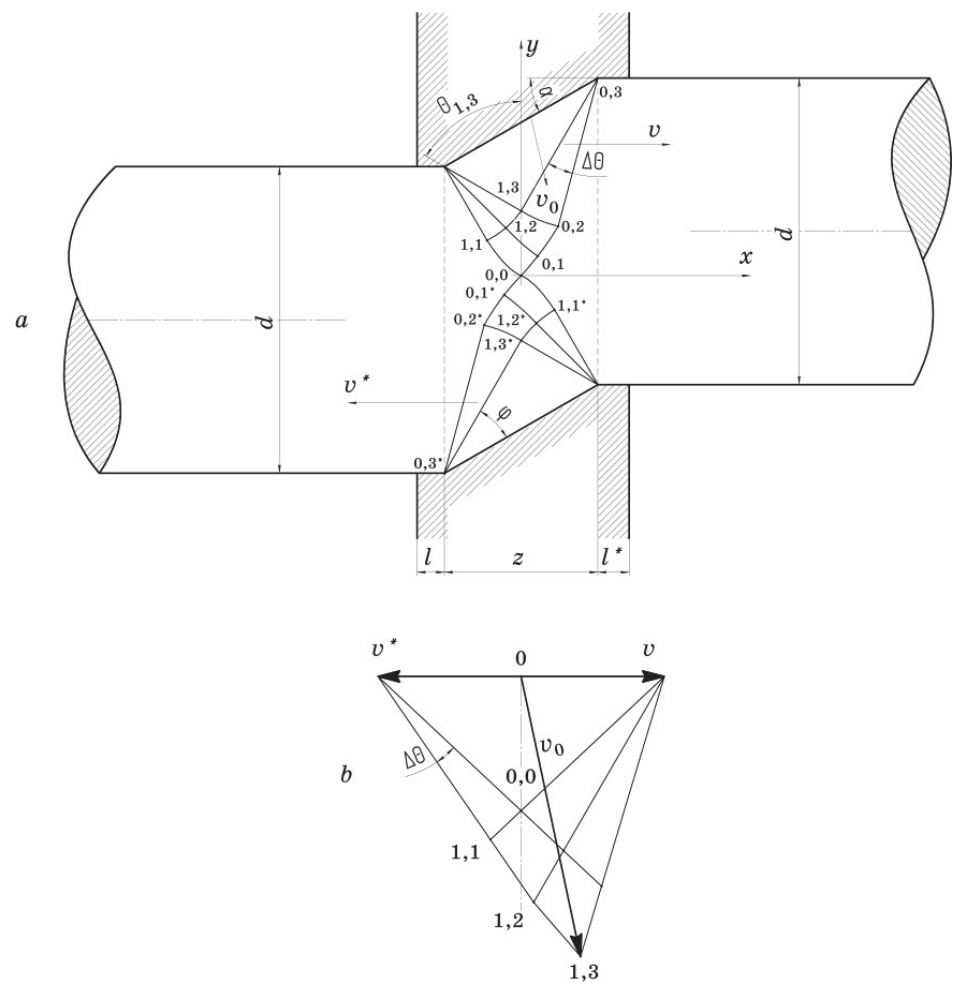

Fig. 4. Slip line field $(a)$ and velocity hodograph $(b)$ on an inclined shear section at $\alpha=30^{\circ}$ and $d / z=2.0$.

Plotting a field of s.l. and the velocity hodograph, as well as the calculation of the stress components were performed according to the above method, i.e. the angle of inclination of the slip lines to the $y$-axis at the nodal point $(1,3)$ is $\theta_{1,3}=60^{\circ}$. Assume the step of changing the s.l. $\Delta \theta=15^{\circ}$, then adding $\Delta \theta$ to the value $\theta_{1,3}$, we obtain the corresponding value of the angle of inclination of the s.l. to the $y$-axis at the nodal point 0,2 , i.e. $\theta_{0,2}=75^{\circ}$. At the nodal point $(1,2)$, the inclination angle of s.l. to the $y$-axis will be $\theta_{1,2}=45^{\circ}$, since we subtract $\Delta \theta$ from the value $\theta_{1,3}$. At point $(1,1)$, the angle of inclination of s.l. to the $y$ axis will be $\theta_{1,1}=30^{\circ}$, etc. The construction was carried out before crossing the s.l. with the $x$-axis, which 
is intersected by s.l. at an angle of $45^{\circ}$, which indicates the correctness of the solutions obtained. Next, a kinematic possible velocity field is constructed (see Fig. 4b), where from the incompressibility condition we can write:

$\frac{v_{0}}{v}=\frac{d}{Z}=2.0$

where $v_{0}$ and $v$ are the metal flow rate along the $y$ and $x$ axes, $d$ is the diameter of the workpiece in the zone of plastic deformation, $z$ is the section of shear of one part of the workpiece relative to another (see Fig. 4a). Hence, with an error of $\sim 1-3 \%$, it can be assumed that the incompressibility condition is fulfilled, i.e. the constructed field of s.l. corresponds to the kinematically possible velocity fields under known boundary conditions. According to the above methodology, the mean stress and stress components were calculated at the central nodal point $(0,0)$. After the appropriate transformations:

$\frac{\sigma_{0,0}}{2 k}=-0.146$

Stress components at the axial nodal point 0,0 :

- stress along the $x$-axis:

$\sigma_{x_{0,0}}=\sigma_{0,0}+k \sin 2 \cdot \theta_{0,0}=-2 k(0.146+0.5)=-2 k \cdot 0.646$,

- stress along the $y$-axis:

$\sigma_{y_{0,0}}=\sigma_{0,0}-k \sin 2 \cdot \theta_{0,0}=-2 k(0.146-0.5)=2 k \cdot 0.354$

- shear stress at the axial nodal point 0,0 :

$\tau_{x y_{0,0}}=-k \cos 2 \cdot \theta_{0,0}=0$.

The results of calculating the stress values at the nodal points of the mesh of s.l. are shown in Table 1 .

Table 1. Values of stress components at nodal points of the slip line field when pulling workpieces in a special die.

\begin{tabular}{cccccccc}
\hline & \multicolumn{3}{c}{$\begin{array}{c}\text { Nodal points of slip line fields at } \alpha \\
=20^{\circ}, d / z=1.5 \text { and } f=0.321\end{array}$} & \multicolumn{4}{c}{$\begin{array}{c}\text { Nodal points of slip line fields at } \\
\alpha=30^{\circ}, d / z=2.0 \text { and } f=0.25\end{array}$} \\
\cline { 2 - 8 } \begin{tabular}{c} 
Stress values, $\mathrm{MPa}$ (multiplied by $2 k$ ) \\
\cline { 2 - 8 }
\end{tabular} & 0,0 & $\begin{array}{c}0,1 \text { and } \\
1,1^{*}\end{array}$ & 1,2 & 0,0 & $\begin{array}{c}0,1 \text { and } \\
1,1^{*}\end{array}$ & 0,2 & 1,3 \\
\hline$\sigma_{0,0}$ & -0.947 & -1.268 & -0.947 & -0.146 & -0.408 & -0.670 & -0.408 \\
$\sigma_{y}$ & -1.447 & -1.701 & -1.447 & -0.646 & -0.841 & -0.920 & -0.908 \\
$\sigma_{x}$ & -0.447 & -0.835 & -0.447 & 0.354 & 0.025 & -0.420 & -0.092 \\
$\tau_{x y}$ & 0 & $\begin{array}{c}0.288 \text { and } \\
-0.288\end{array}$ & 0 & 0 & $\begin{array}{c}0.288 \text { and } \\
-0.288\end{array}$ & 0.500 & 0 \\
\hline
\end{tabular}

2.3.3 Study of the effect of contact friction between the working surface of the die channel and the workpiece on the stress state and pulling force

In addition to the angle of inclination of the working surface $\alpha$ and the ratio $d / z$, the value of contact friction $f$ has a significant effect on the stress state and pulling force. Therefore, in order to study the influence of contact friction on the distribution of stress and pulling force, a field of s.l. was constructed and the hodograph of velocities at $f=0$, shown in Fig. 5. 


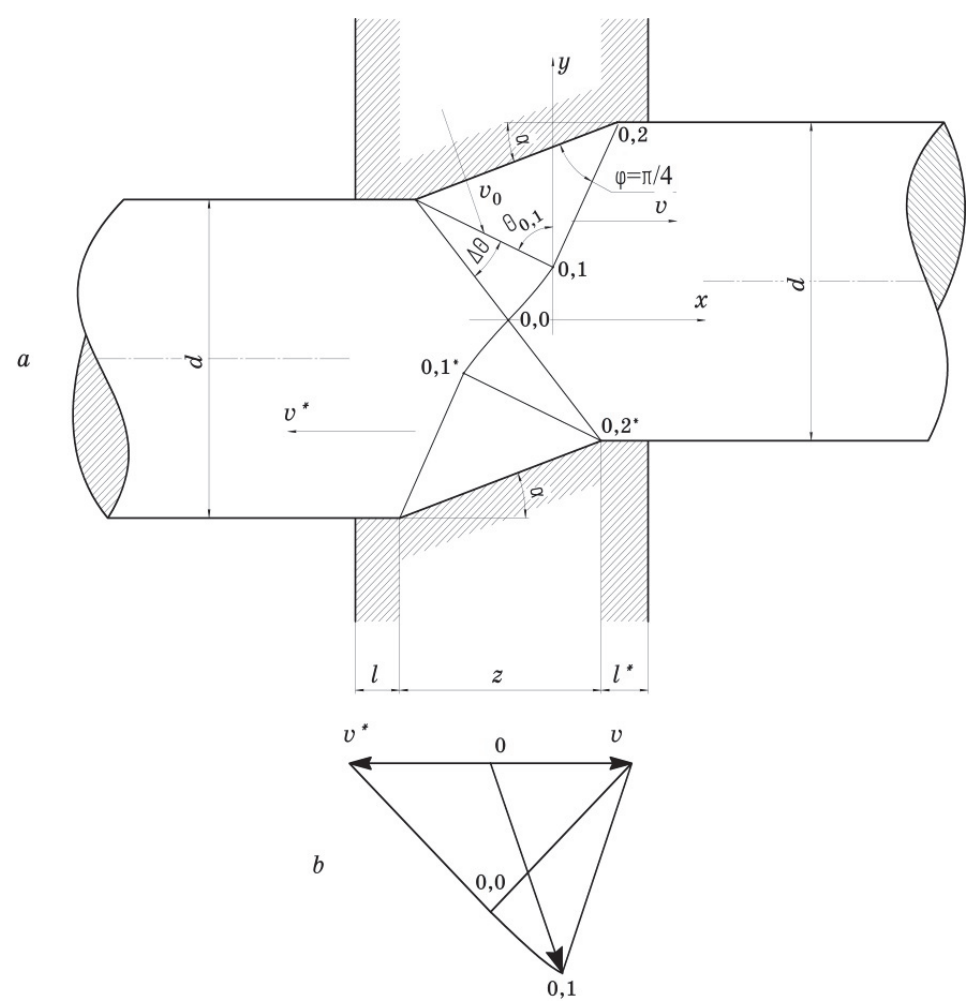

Fig. 5. Slip line field $(a)$ and velocity hodograph $(b)$ on an inclined shear section at $\alpha=20^{\circ}, d / z=1.5$ and at a friction coefficient $f=0$.

It is known that at $f=0$ the s.l. come out to the contact surface at an angle of $45^{\circ}$. Constructing a field s.l. and the hodograph of velocities was carried out according to the above method, using the basic properties of s.l. The mean normal stress at the nodal point $(0,0)$ was determined from the condition of the equilibrium of forces applied to the plastic zone on the right:

$$
\int_{0,0}^{0,1} \sigma d y+\sigma_{0,1}\left(y_{0,2}-y_{0,1}\right)+k x_{0,2}+\sigma_{0,0}\left(y_{0,0}-y_{0,2^{*}}\right)+k x_{0,2^{*}}=0
$$

Using Hencky equations (Dixit \& Dixit, 2014; Hill, 1998; Yu et al., 2006; Rees, 2006), after substitution into the above equation and the corresponding transformation:

$\frac{\sigma_{0,0}}{2 k}=-0.110$

As in the previous cases, compressive stresses act mainly in the zone of plastic deformation of the workpiece (Table 2).

Table 2. Values of stress components at nodal points of the slip line field when pulling workpieces in a special die at $f=0$.

\begin{tabular}{ccc}
\hline $\begin{array}{c}\text { Stress values, MPa } \\
\text { (multiplied by } 2 k)\end{array}$ & Nodal points of slip line fields at $\alpha=20^{\circ}$ и $d / z=1.5$ & 0,1 \\
\hline$\sigma_{0,0}$ & 0,0 & -0.460 \\
$\sigma_{y}$ & -0.110 & -0.843 \\
$\sigma_{x}$ & -0.610 & -0.077 \\
$\tau_{x y}$ & 0.390 & 0.942 \\
\hline
\end{tabular}


However, the stress values, as expected, are 8 times less than the corresponding stress values obtained when pulling workpieces in dies with an inclination angle $\alpha=20^{\circ}, d / z=1.5$, and a friction coefficient $f=0.321$. The values of the mean stress in comparison with the corresponding mean stress obtained by pulling the workpieces in the dies with an inclination angle $\alpha=30^{\circ}, d / z=2.0$ and a friction coefficient $f=0.25$ are 1.3 times less.

Table 2 shows that the axial tensile stress has a minimum value $(2 k \cdot 0.39)$, and closer to the contact surface transforms into a compressive one. The specific pulling force was determined through the normal stress $\sigma_{n}$ at the nodal point 0,1 , which is 1.7 times less than the corresponding value of the specific force obtained at $\alpha=20^{\circ}, d / z=1.5$, and the friction coefficient $f=0.321$. It should be noted that in all considered variants of pulling processes in a special die, plastic deformation covers or spreads only in the shear section of the workpiece $z$. In this case, the diameter of the workpiece $d$ at the entrance and exit from the deformation zone does not change, and the s.l. field and the hodograph of velocities can be considered built correctly.

\section{Results and discussion}

The analysis of the obtained calculation results when pulling the workpieces in a special die with the angles of inclination of the working surface $\alpha$ equal to $20^{\circ}$ and $30^{\circ}$, as well as with the values of the $d / z$ ratio equal to 1.5 and 2.0, respectively, show that in the zone of plastic deformation, i.e. significant compressive stresses (with a minus sign) act mainly at the nodal points of the slip line field. In this case, the stress values when pulling the workpieces do not pose any danger, since they are compressive and will create a significant obstacle to the stretching of grains, destruction of the metal in the direction of pulling and contribute to the closure and removing of all internal defects of the workpieces. Fig. 6 shows the results of calculating the mean normal stresses at the central nodal points $(0,0)$ for different values of the angle of inclination or shear angle $\alpha$ of the working channel of the die. The decrease in the mean normal stresses at the central nodal points 0,0 , depending on the change in the angle $\alpha$, can be explained as follows. A decrease in the mean normal stresses $\sigma_{0,0}$ is primarily due to the fact that with a decrease in the angle $\alpha$, all forces are mainly concentrated in the central axial zone of the workpiece. Therefore, all stresses, including mean stresses in the axial zone increase. A completely different picture is observed with increasing angle $\alpha$. With an increase in angle $\alpha$, the forces shear more and more into the horizontal plane, thereby reducing the normal stresses in the axial zone and increasing the shear stresses more and more, which is quite natural, since with an increase in the angle $\alpha$, shear strain develops. For example, when open-die forging in flat dies (at $\alpha=0$ ), all forces are concentrated in the axial central zone and, depending on the $d / z$ ratio, tensile normal stresses prevail mainly in the deformation zone. From this point of view, it is advisable to take the average value of the angle of inclination $\alpha=20^{\circ}$, since with decreasing angle $\alpha$, the total pulling force decreases and the mean normal stress in the axial zone of the deformation zone increases, which prevents the stretching of grains in the pulling direction and reduces the probability of metal fracture in this direction.

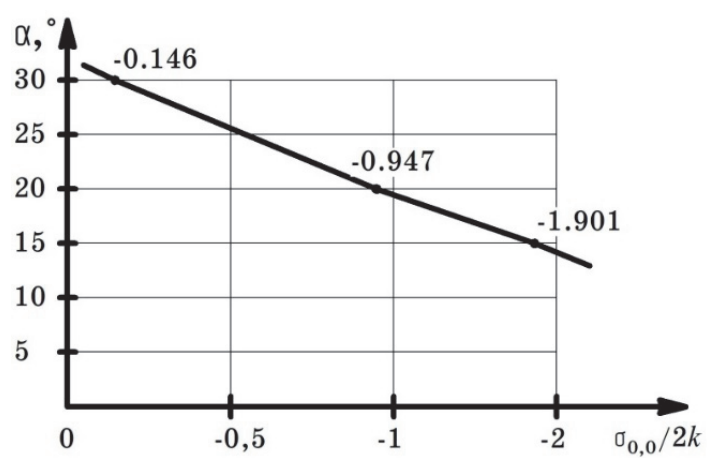

Fig. 6. The change in the mean stress at the central nodal point $(0,0)$ depending on the angle of inclination of the shear $\alpha$ of the die 
Changes in shear stresses show that at first they decrease to the nodal point $(0,0)$, change sign when passing through this point 0,0 , then increase, causing compression in the diagonal directions of the deformation zone of the workpiece. In this case, from the side of the die protrusion (at point 1,1) it is compressive, and in the direction of the velocity $v$ (at points 0,1 and 0,2 ) it is tensile, which is quite true, since the compressed metal is displaced in this direction. It can be seen that it in this small diagonal direction, i.e. along the s.1. 2.2-1.1-0.0 and $0.0-1.1^{*}-2.2^{*}$, shear fracture is also possible with a relatively small gap $z$ and maximum values of shear stresses $\tau_{x y}$. Shear stresses are distributed more evenly than normal stresses. A decrease in the values of normal stresses at $\alpha=30^{\circ}$ and $d / z=2.0$ is also associated with an increase in the relative height of the shape of the zone of plastic deformation, i.e. with increasing $d / z$ ratio. At relatively high shapes of the deformation zone, as is known from the theory of metal forming, tensile stresses develop at the axial central point. A similar case is observed here, where tensile stresses appear at the axial point 0,0 during pulling in dies with $\alpha=30^{\circ}$ and the ratio $d / z=2.0$ (see Table 1), which turn into compressive stresses closer to the contact surface. Hence, it can be seen that the larger the ratio (at $d / z \geq 2.0$ ) or the shape of the zone of plastic deformation, the greater is not only the probability of the indention of the protrusion of the die and shear fracture of the workpiece, but also the appearance of tensile stresses in the axial zone, contributing to the elongation of the metal structure or destruction metal. When pulling a low-plasticity metal and a further increase in these values, shear fracture of the workpiece may occur. On the contrary, at lower values of the angle of inclination $\alpha$ and a relatively low shape of the zone of plastic deformation, i.e. at $d / z<2.0$, all stress values do not pose any danger, since they are all compressive, and cannot lead to stretching of grains and cracking in the metal in the direction of pulling.

The strain resistance from the side of the inclined surface or the pulling force $P_{p u l}$ can be determined through the normal stresses acting along the $y$-axis at the nodal points $(1,2)$ and $(1,3)$, respectively, when pulling the workpieces in a special die with the tilt angles of the working surface $\alpha=20^{\circ}$ and $\alpha=30^{\circ}$, ratio $d / z=1.5$ and $d / z=2.0$ according to the following formula:

$$
P_{p u l}=\sigma_{y_{0,0}} \tan \alpha F_{c}
$$

where $\alpha$ is the angle of inclination of the working channel of the die, $F_{c}$ is the contact area of the inclined surface of the protrusion of the die and the workpiece. The results of calculating the pulling force show that the values of the pulling force in dies with $\alpha=30^{\circ}$ and $d / z=2.0$ are 1.5 times greater than at $\alpha=20^{\circ}$ and $d / z=1.5$. Hence, the assumption is confirmed that the angle of inclination $\alpha$ cannot be increased, since slip of the rolling mill rolls in front of the die may occur due to the high resistance from the wall of the inclined die surface.

It should be noted that an increase in the values of all stresses in the deformation zone in dies with $\alpha$ $=20^{\circ}$ and $d / z \approx 1.5$ can be explained by the effect of increasing the pressure in the liquid with a decrease in the cross-sectional area of the flow. Roughly, the same thing happens when the workpieces are pulled through a special die, where at the same $z$ value and when $d$ changes, the stress state and power parameters change.

Stress values decrease at minimum contact friction values. However, it must be taken into account that it is very difficult to create conditions for reducing contact friction, especially when hot pulling in a special die. Thus, the results of the analysis of the stress state and force parameters show that when pulling workpieces with a large ratio $d / z>2.0 \div 3.0$ in dies with a large angle of inclination $\left(\alpha=45^{\circ}\right)$, the upper protrusion of the tool will be indented into workpiece, and the shear may not occur. When pulling workpieces with a ratio of $d / z \geq 2.0$ in dies with an angle of inclination $\alpha=30^{\circ}$, tensile normal stresses appear in the plastic deformation zone, contributing to rupture and elongation of the metal microstructure. In addition, with a further increase in the $d / z$ ratio or a decrease in the gap $z$, there is a possibility of separation of one part of the workpiece from another. It should be noted that it is impractical to reduce 
this $d / z$ ratio, since at small values, the length of the inclined surface of the die protrusion increases, and this can lead to an increase in the resistance of the forces of contact friction and the total pulling force. Therefore, in order to avoid the elongation of grains, destruction of the metal and the appearance of large pulling forces, a special die should be chosen with an angle of inclination of the working surfaces $\alpha=$ $20^{\circ}$ with a ratio $d / z \approx 1.5$. The use of a special die with these parameters after the next pass of the workpiece between the rolling mill rolls or passing through the channel of the drawing die can provide a more homogenous structure of the metal by changing and preventing the unidirectional flow of the metal and prevent cracking in the direction of pulling. Based on the results obtained, a special die with a displaced upper inclined working surface has been developed and preparatory work is underway for conducting experimental studies. A special die is supposed to be installed after a round pass of a rolling mill (Naizabekov et al., 2010) or a radial-shear rolling mill (Mashekov et al., 2020), and thereby create additional shear strain in the longitudinal directions. The rolling mill implements intensive rolling in the rhombus-square-circle passes system, where, in contrast to traditional rhombic passes, rhombic passes with an off-diagonal arrangement will be used (Naizabekov et al., 2010), which provide transverse shear. The radial shear mill of a new design (Mashekov et al., 2020) also provides intensive metal working.

\section{Conclusions}

The use of a special die with an angle of inclination of the working surfaces $\alpha=20^{\circ}$ with a ratio $d / z$ $\approx 1.5$, in the composition of section, wire and radial shear mills contributes to the creation of a more favorable SSS scheme, providing a more homogeneous metal structure (up to obtaining ultrafine-grained and/or nanostructures) throughout the volume of the workpiece, due to a change in the nature of the metal flow in the longitudinal direction of pulling. The stress distribution in the zone of plastic deformation depends on the angle of inclination $\alpha$ and the ratio of the dimensions of the shape of the zone of plastic deformation, i.e. on the $d / z$ ratio. For too large values of $\alpha\left(\right.$ at $\left.\alpha=45^{\circ}\right)$ and $d / z$ ratios $($ at $d / z \geq 2.0)$, the insertion of the die protrusions into the workpiece or cut is possible, as well as the appearance of tensile stresses in the axial zone. At small values of the $d / z$ ratio, the total length of the inclined surface increases, and, accordingly, this can lead to an increase in the resistance of the forces of contact friction. In addition, the pulling force increases with increasing die angle $\alpha$. Therefore, the optimal $d / z$ ratio is $d / z=1.5$ and the angle of inclination of the working channel of the die $\alpha=20^{\circ}$. With such ratios, the workpiece is maximally displaced, as a result of which the axial tensile stresses decrease, which contributes to a more uniform distribution of mechanical properties and the formation of a more uniform structure throughout the volume of the pulled workpiece.

\section{References}

Abishkenov, M., Ashkeyev, Zh., Mashekov, S., Akhmetova, G., \& Volokitina, I. (2020). Investigation of the stress-strain state of balls under deformation in a closed die. Metalurgija, 59(4), 559-562.

Ashkeev, Zh., Naizabekov, A., Lezhnev, S., \& Toleuova, A. (2005). Billet deformation in uniformchannel stepped die. Steel in Translation, 35(2), 37-39.

Azushima, A., Kopp, R., Korhonen, A., Yang, D.Y., Micari, F., Lahoti, G.D., Groche, P., Yanagimoto, J., Tsuji, N., Rosochowski, A., \& Yanagida, A. (2008). Severe plastic deformation (SPD) for metals. CIRP Annals - Manufacturing Technology, 57(2), 716-735.

Bhaduri, A. (2006). Mechanical Properties and Working of Metals and Alloys. Berlin-Heidelberg: Springer.

Carlsson, B., \& Huml, P. (1996). Determination of the Material Properties of an Anisotropic Metal Wire. CIRP Annals, 45(1), 231-233.

Chung, K. \& Lee, M.-G. (2018). Basics of Continuum Plasticity. Singapore: Springer.

Dixit, P.M. \& Dixit, U.S. (2014). Plasticity: Fundamentals and Applications. Boca Raton: CRC Press.

Doltsinis, I. (2001). Elements of Plasticity: Theory and Computation. Southempton: WIT Press.

Frint, P., \& Wagner, M. (2019). Strain partitioning by recurrent shear localization during equal-channel angular pressing of an AA6060 aluminum alloy. Acta Materialia, 176, 306-317. 
Ghader, F., Hyoung S.K., \& Hessam, T.K. (2018). Severe Plastic Deformation: Methods, Processing and Properties. Amsterdam: Elsevier.

Goloujeh, M.R., \& Soltanpour, M. (2020). Simple Shear Forging as a Method for Severe Plastic Deformation. International Journal of Lightweight Materials and Manufacture (in press, journal preproof).

Hardy, C., Baronet, C.N., \& Tordion, G.V. (1971). The elasto-plastic indentation of a half-space by rigid sphere. International Journal for Numerical Methods in Engineering, 3(3), 451-462.

Harsha, R.N., Mithun Kulkarni, V., \& Satish Babu, B. (2018). Severe Plastic Deformation - A Review. Materials Today: Proceedings, 5(10), 22340-22349.

Hill, R. (1998). The Mathematical Theory of Plasticity. Oxford: Oxford University Press.

Hosford, W.F. (2013). Fundamentals of Engineering Plasticity. New York: Cambridge University Press.

Iordachescu, M., de Abreu, M., \& Valiente, A. (2015). Effect of cold-drawn induced anisotropy on the failure of high strength eutectoid and duplex steel wires. Engineering Failure Analysis, 56, 412-421.

Lee, C.H., \& Kobayashi, S. (1970). Elastoplastic analysis of plane strain and axisymmetric flat punch indentation by the finite element method. International Journal of Mechanical Sciences, 12(4), 349370.

Mashekov, S., Angarbekov, U., Uderbayeva, A., \& Sarsenbayev, N. (2020). New-design radial-shear rolling mill (RSRM) automation. Metalurgija, 59(4), 563-566.

Naizabekov, A., Bykhin, M., Nogaev, K., \& Bykhin, B. (2010). Study of the process of realization of high-rate plastic deformation in lengthwise rolling. METAL 2010 - 19th International Conference on Metallurgy and Materials, Conference Proceedings (Roznov pod Radhostem: Tanger Limited),192202.

Rahimi, F., Eivani, A.R., Jafarian, H.R., \& Bhattacharjee, T. (2017). Effect of pure shear strain on mechanical properties and microstructural evolution. Materials Science and Engineering: A, 679, $133-142$.

Rajan, K., \& Petkie, R. (1998). Microtexture and anisotropy in wire drawn copper. Materials Science and Engineering: A, 257(1), 185-197.

Rees, D.W.A. (2006). Basic Engineering Plasticity: An Introduction with Engineering and Manufacturing Applications. Oxford: Butterworth-Heinemann.

Segal, V.M. (1999). Equal channel angular extrusion: from macromechanics to structure formation. Materials Science and Engineering: A, 271(1-2), 322-333.

Toribio, J. (2019). Crack path deflection in cold-drawn pearlitic steel as a consequence of microstructural anisotropy generated by manufacturing: Resembling Picasso, Larionov and Goncharova. Procedia Structural Integrity, 16, 281-286.

Valiev, R.Z., Zhilyaev, A.P., \& Langdon, T.G. (2014). Bulk Nanostructured Materials: Fundamentals and Applications. Hoboken, New Jersey: John Wiley \& Sons, Inc.

Voyiadjis, G.Z., \& Buckner, N.E. (1983). Indentation of a half-space with a rigid indentor. International Journal for Numerical Methods in Engineering, 19(10), 1555-1578.

Wright, R.N. (2016). Wire Technology: Process Engineering and Metallurgy. Oxford: ButterworthHeinemann.

Yu, M.-H., Ma, G.-W., Qiang, H.-F., \& Zhang, Y.-Q. (2006). Generalized Plasticity. Berlin-Heidelberg: Springer.

Zehetbauer, M.J., \& Valiev, R.Z. (2004). Nanomaterials by Severe Plastic Deformation. Hoboken, New Jersey: John Wiley \& Sons, Inc.

Zolotorevsky, N., \& Krivonosova, N. (1996). Effect of ferrite crystals' plastic anisotropy on residual stresses in cold-drawn steel wire. Materials Science and Engineering: A, 205(1-2), 239-246. 
(C) 2021 by the authors; licensee Growing Science, Canada. This is an open access article distributed under the terms and conditions of the Creative Commons Attribution (CC-BY) license (http://creativecommons.org/licenses/by/4.0/). 